\title{
Generating a mass gap using Feynman diagrams in an asymp- totically free theory
}

\author{
Venkitesh Ayyar ${ }^{1}$ and Shailesh Chandrasekharan ${ }^{2, \star}$ \\ ${ }^{1}$ Department of Physics, University of Colorado at Boulder, Boulder CO 80309 \\ ${ }^{2}$ Department of Physics, Duke University, Durham NC 27708
}

\begin{abstract}
Using the example of a two dimensional four-fermion lattice field theory, we show that Feynman diagrams can generate a mass gap in a theory with massless fermions that interact via a marginally relevant coupling. We show this by introducing an infrared cutoff that makes the perturbation series for the partition function convergent. We use a Monte Carlo approach to sample sufficiently high orders of diagrams and thus expose the presence of the mass gap.
\end{abstract}

\section{Introduction}

Asymptotically free quantum field theories are defined through a Gaussian fixed point with a marginally relevant coupling. This suggests that a non-perturbative approach based on an expansion in powers of some bare coupling $U$ should be able to describe the physics quantitatively. Recently, Monte Carlo methods that sample bare weak coupling Feynman diagrams have emerged as a new way to extract non-perturbative physics in a variety of models [1-6]. Can such methods be extended to asymptotically free theories like QCD? Setting aside complications due to gauge invariance, here we explore this question in a simple fermionic toy model in two dimensions. Similar questions have been raised recently and partially addressed within the context of the two dimensional $O(N)$ and $U(N) \times U(N)$ models in the large $N$ limit $[7,8]$. Non-perturbative methods based on resurgence theory [9] and the bootstrap program have also addressed similar questions recently [10].

Naively, one would think that it is impossible to obtain the mass gap using a weak coupling diagrammatic approach, since the non-perturbative mass gap in the theory scales as $M \sim \mathrm{e}^{-\beta / U}$. The essential singularity at $U=0$ would invalidate any perturbative approach. In this talk we argue that by regulating the ultraviolet and infrared divergences in a controllable way, it is possible to make the weak coupling series convergent at least in two dimensional four-fermion field theories like the Gross Neveu models that are known to be asymptotically free [11, 12]. We consider an explicit example of the lattice model and show how Feynman diagrams generate the mass gap in this theory [13].

\footnotetext{
^ Speaker (e-mail: sch@phy.duke.edu) This work was supported by U.S. Department of Energy, Office of Science, Nuclear Physics program under Award Number DE-FG02-05ER41368.
} 


\section{Lattice Model}

The model we consider is constructed using reduced staggered fermions [14, 15], whose action is given by

$$
S(\psi)=\frac{1}{2} \sum_{x, y, a} \psi_{x}^{a} M_{x, y} \psi_{y}^{a}-U \sum_{x} \psi_{x}^{4} \psi_{x}^{3} \psi_{x}^{2} \psi_{x}^{1},
$$

where $M_{x, y}$ is the free staggered fermion matrix

$$
M_{x, y}=\frac{1}{2} \sum_{\alpha} \eta_{\alpha, x}\left(\delta_{x+\hat{\alpha}, y}-\delta_{x-\hat{\alpha}, y}\right),
$$

with the phase factors $\eta_{1, x}=1, \eta_{2, x}=(-1)^{x_{1}}$. Note that there is only a single Grassmann valued field $\psi_{x}^{a}$ in the lattice action. The partition function of the model is given by

$$
Z=Z_{0} \int[d \psi] \mathrm{e}^{-S(\psi)}
$$

where $Z_{0}$ is chosen so that $Z=1$ in the free theory and the integration measure $[d \psi]$ is the propduct of $\left[d \psi_{x}^{1} d \psi_{x}^{2} d \psi_{x}^{3} d \psi_{x}^{4}\right]$ on every site $x$.

At $U=0$ our lattice model describes four flavors of free massless (two-component) Dirac fermions in the continuum limit, which is reached by exploring physics at large length scales as compared to the lattice spacing. As a probe of the long distance physics we can take space-time to be a torus of side $L$ (in lattice units) in each direction with anti-periodic boundary conditions. In two dimensions, a free fermion field is expected to have a mass dimension $\left[\psi_{x}^{a}\right]=1 / 2$. In our approach this can be seen from the scaling of the fermion propagator $G_{f}(x, y) \sim 1 /|x-y|$ for large separations. In Fig. 1 we plot the scaling of the propagator at a separation of $|x-y|=L / 2$ along one of the directions, $R=G_{f}(0, L / 2)$ as a function of $L$. As expected we find $R \sim 1 / L$. In the same figure we also show the scaling of the susceptibility

$$
\chi_{1}=\frac{1}{2 Z} \int[d \psi] \mathrm{e}^{-S} \sum_{y}\left\{\psi_{0}^{a} \psi_{0}^{b} \psi_{y}^{b} \psi_{y}^{a}\right\}
$$

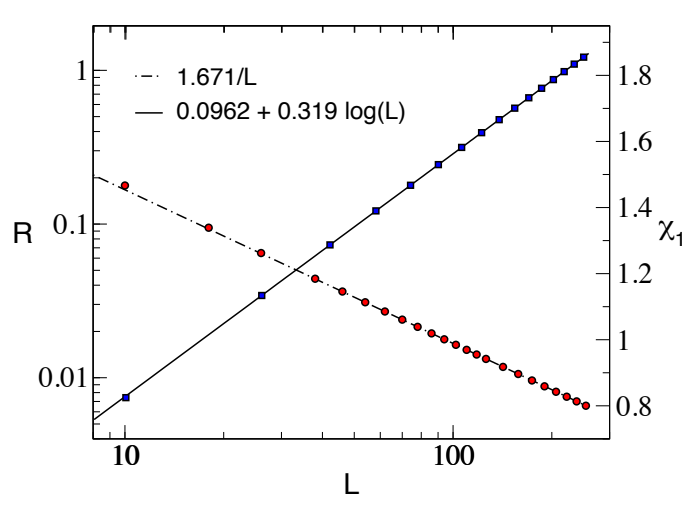

Figure 1. The scaling of the fermion propagator at the mid point $(R)$ and the susceptibility $\left(\chi_{1}\right)$ as a function of $L$ in the free theory. This shows that the coupling $U$ is perturbatively marginal as expected in the continuum. 
as a function of $L$. This susceptibility is naively dimensionless, but due to infrared singularities it is expected to diverge logarithmically with the lattice size $L$. This behaviour is consistent with the fact that the coupling $U$ is marginal as expected from continuum perturbative power counting. We will see later that in fact $U$ is marginally relevant since an exponentially small mass gap is generated in this model when $U>0$ due to asymptotic freedom as predicted originally by Gross and Neveu [11].

\section{The Partition Function}

Let us first expand the partition function of our model in powers of the coupling,

$$
\begin{aligned}
Z & =Z_{0} \int[d \psi] \mathrm{e}^{-\frac{1}{2} \psi^{T} M \psi} \mathrm{e}^{\sum_{x} U \psi_{x}^{4} \psi_{x}^{3} \psi_{x}^{2} \psi_{x}^{1}}=Z_{0} \int[d \psi] \mathrm{e}^{-\frac{1}{2} \psi^{T} M \psi} \prod_{x}\left(1+U \psi_{x}^{4} \psi_{x}^{3} \psi_{x}^{2} \psi_{x}^{1}\right) \\
& =Z_{0} \sum_{k} U^{k} \sum_{[x, k]} \prod_{a}\left(\int\left[d \psi^{a}\right] \mathrm{e}^{-\frac{1}{2} \psi_{x}^{a} M_{x, y} \psi_{y}^{a}} \psi_{x_{1}}^{a} \psi_{x_{1}}^{a} \ldots \psi_{x_{k}}^{a}\right)=\sum_{k} U^{k} z_{k},
\end{aligned}
$$

where the coefficients $z_{k}$ can be obtained as a sum over weights of all possible vertex configurations $[x ; k]=\left\{x_{1}, x_{2}, \ldots, x_{k}\right\}$. Each configuration is an ordered set of $k$ different lattice sites where the interactions occur and has the weight given by

$$
\Omega([x ; k])=Z_{0} \prod_{a}\left(\int[d \psi] \mathrm{e}^{-\frac{1}{2} \psi_{x}^{a} M_{x, y} \psi_{y}^{a}} \psi_{x_{1}}^{a} \psi_{x_{2}}^{a} \ldots \psi_{x_{k}}^{a}\right)^{4}=\left(\operatorname{Pf}(W([x ; k]))^{4},\right.
$$

which is a sum over Feynman diagrams obtained through the regular Wick contractions. In the last step we have used the fact that for each flavor the sum over the diagrams is equal to the Pfaffian of a $k \times k$ matrix $W([x ; k])$, whose matrix elements are given by the free staggered fermion propagator $G_{f}\left(x_{i}, x_{j}\right)$ between the sites in $[x ; k][16]$. Since $\Omega([x ; k])$ is positive a Monte Carlo method can then be used to sample the vertex configurations $[x ; k]$, which is distributed according to the probability distribution

$$
P_{k}(U,[x ; k])=\frac{U^{k}}{Z(U)} \Omega([x ; k]) .
$$

Due to symmetries of the model only configurations $[x ; k]$ with an equal number of even and odd sites have non-zero weights, which implies that only even values of $k$ contribute to the expansion in Eq.(5).

Interestingly, the expansion in powers of $U$ is finite and completely convergent on a finite lattice. Since the maximum number of vertices that are allowed is $L^{2}$ the partition function $Z$ is a polynomial. Our goal is to understand how the infrared divergences present in an asymptotically free theory manifest themselves in this polynomial. In order to gain some insight into the dominant terms in the expansion we define the probability distribution of vertices, $P_{k}(U)=z_{k} U^{k} / Z(U)$. Note that $P_{k}(U)$ as the sum over $P_{k}(U,[x, k])$ with a fixed $k$ and $U$ but different locations of the vertices. While it is difficult to compute $P_{k}(U)$ analytically we can use Monte Carlo sampling to estimate it. In Fig.2(a) we plot $P_{k}(U)$ at $U=0.1$ for different values of $L$. Note that sectors with a large number of vertices are suppressed exponentially and the average number of vertices is much smaller than the maximum allowed value of $k_{\max }=L^{2}$. Instead of the average number of vertices, a more physically interesting quantity is the average density of vertices $\rho(U)=\langle k\rangle / L^{2}$. In Fig. 2(b) we show how $\rho(U)$ changes with $U$. In the inset of Fig. 2(b) we plot the density at $U=0.1$ for various lattice sizes and observe that it rapidly saturates in the thermodynamic limit. Thus, the physics of the model at a fixed $U$ is described by Feynman diagrams that contain a fixed density of vertices. At $U=0.1$, the average density is very small $\rho=0.0027$. 

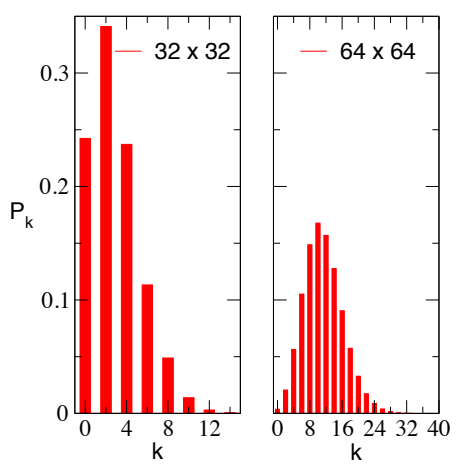

(a)

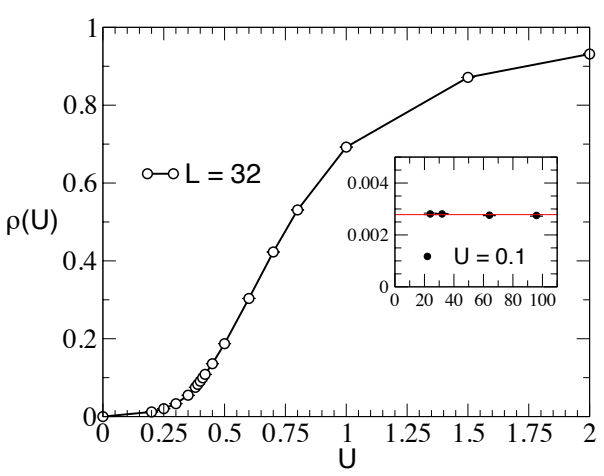

(b)

Figure 2. (a) Probability distribution of vertices in the partition function as a function of the lattice size at $U=0.1$ for $L=32,64$ and 96 . The average density of vertices $\rho_{k}=\langle k\rangle / L^{2} \approx 0.00267$ remains constant in all the three cases. (b) Plot of the density of vertices $\rho(U)$ as a function of $U$. The inset shows the density at $U=0.1$ as a function of $L$. We see that the density of vertices remains the same as $L$ increases.

It is easy to understand why the average density of vertices is constant in the thermodynamic limit. From the point of view of statistical mechanics one expects the partition function of a two dimensional system to scale as $Z=\exp \left(f(U) L^{2}\right)$, where $f(U)$ is the free energy density. Interestingly, the average density of vertices is related to $f(U)$ through the expression

$$
\rho(U)=\frac{\langle k\rangle}{L^{2}}=\left(U / L^{2}\right)(\partial \ln Z(U) / \partial U)=U(\partial f(U) / \partial U) .
$$

Since $f(U)$ is expected to be independent of the volume for sufficiently large volumes, we also expect $\rho(U)$ to be independent of the volume.

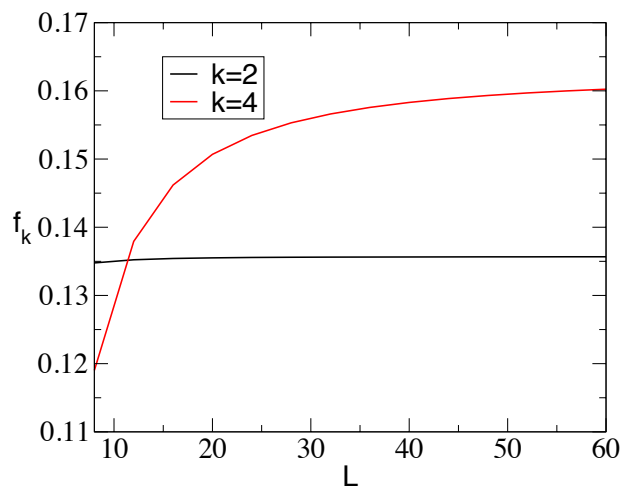

Figure 3. Plot of the perturbative coefficients $f_{2}$ and $f_{4}$ in the expansion of the free energy as a function of $L$.

The connection between $Z(U)$ and $f(U)$ is well known in diagrammatic perturbation theory; the former contains contributions from disconnected diagrams, while the latter gets contributions only 
from connected diagrams. At a fixed $L$ we can expand $f(U)=f_{2} U^{2}+f_{4} U^{4}+\ldots$ and find relations between the coefficients $f_{k}$ and $z_{k}$. For example $f_{2}=z_{2} / L^{2}$ and $f_{4}=\left(z_{4}-z_{2}^{2} / 2\right) / L^{2}$ and so on. From the discussion above we learn that $Z(U)$ contains no infrared divergences except for the usual factors of the volume, but we cannot rule out such divergences in the expansion of $f(U)$. In general infrared divergences of perturbation theory appear in the $f_{k}$ 's as $L$ is increased. In our model such divergences do not seem to appear at least in the first two terms $f_{2}$ and $f_{4}$ as can be seen in 3 , where these coefficients are plotted as functions of $L$. This seems to be a special feature of our model due to its symmetries. Importantly, even if the $f_{k}$ 's contained divergences we can still extract $f(U)$ nonperturbatively through the integral

$$
f(U)=\int_{0}^{U} \rho(U) / U,
$$

if we can compute $\rho(U)$ non-perturbatively by summing over the distribution of vertices generated by the Monte Carlo method. The usual infrared divergences in perturbation theory disappear after this re-summation.

\section{The Mass Gap}

In order to see how Feynman diagrams generate the mass gap in our model we focus on the finite size susceptibility $\chi_{1}$ defined in (4). As we already pointed out earlier, in the free theory $\chi_{1}$ diverges logarithmically for large values of $L$ (see Fig. 1). However, if a mass gap $M$ is generated we expect $\chi_{1}$ to level off roughly around $L \sim M^{-1}$. The calculation of $\chi_{1}$ can also be expressed as a sum over Feynman diagrams through the relation,

$$
\chi_{1}=\sum_{y, k}\left(\sum_{[x ; k]} \Gamma_{0, y}([x ; k]) P_{k}(U,[x ; k])\right)
$$

where $\Gamma_{0, y}([x ; k])$ is the ratio of two quantities. The numerator is the sum over all Feynman diagrams with two fermion bilinear sources $\psi_{0}^{a} \psi_{0}^{b}$ and $\psi_{y}^{b} \psi_{y}^{a}$, the former located at the origin and the latter at $y$, in addition to the vertices $[x ; k]=\left\{x_{1}, x_{2}, \ldots, x_{k}\right\}$. The denominator is $\Omega([x ; k])$, i.e., the sum over Feynman diagrams without the sources. This ratio makes $\Gamma_{0, y}([x ; k])$ scale like a "connected" Feynman diagram for large volumes since the factor that scales exponentially in the volume is cancelled between the numerator and the denominator.

In order to compute $\chi_{1}$ we generate the vertex configurations $[x ; k]$ with probability $P_{k}(U,[x ; k])$ and then introduce sources, one chosen at a random lattice site which is defined as the origin and another at the site $y$ which is moved sequentially through the lattice. We then compute $\chi_{1}$ using Eq. (10). In Fig. 4(a) we plot $\chi_{1}$ as a function of $L$ at $U=0.3$ and 0.4. Note that indeed $\chi_{1}$ begins to level off around $L \sim 128$ at $U=0.3$ and around $L \sim 32$ at $U=0.4$, implying that a mass gap has been generated. The fact that it takes a substantially larger value of $L$ before $\chi_{1}$ levels off at $U=0.3$ as compared to $U=0.4$ is an indication that $M$ is decreasing rapidly. We also plot the $U=0$ results for comparison.

Statistically speaking this implies that for most vertex configurations $[x ; k]$ generated in the Monte Carlo sample, $\Gamma_{0, y}([x ; k])$ begins to decay exponentially for points $y$ far from the origin. This implies that the infrared divergence of the usual perturbation theory disappears for sufficiently large lattices when we take into account a constant density of vertices. This suggests that we will need to consider large orders of perturbation theory before the infrared divergences will disappear. What about divergences that we do know exist even at small orders of perturbation theory? For example in Fig. 4(a) we see enhancement in $\chi_{1}$ at small values of $L$ due to such divergences. We believe they become statistically insignificant at larger values of $L$. In other words they should be observed as rare but large 

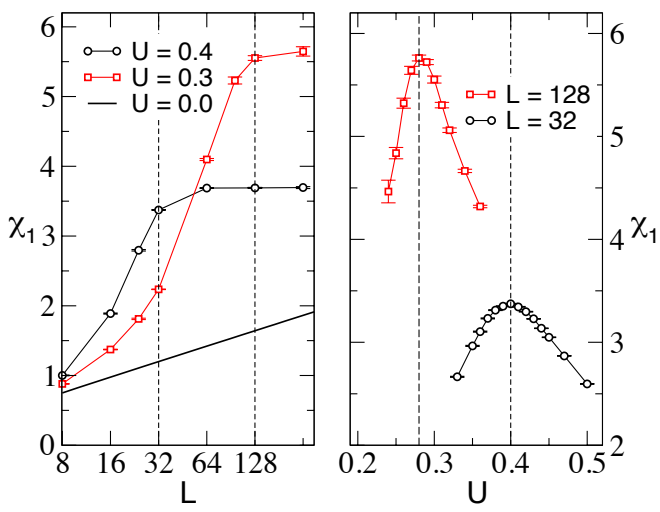

(a)

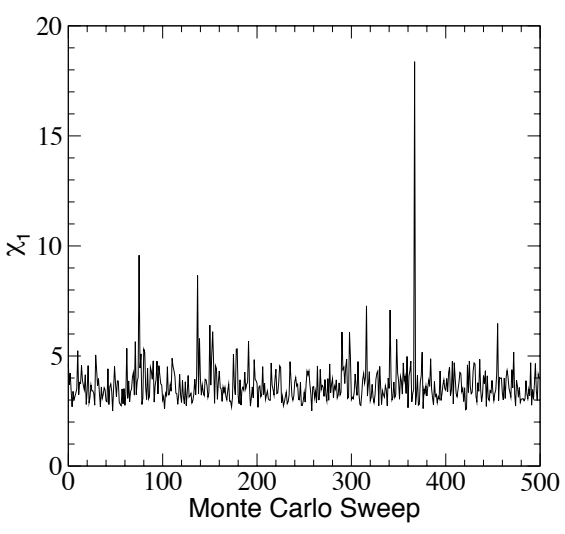

(b)

Figure 4. (a) Plot of the susceptibility $\chi_{1}$ as a function of $L$ for $U=0,0.3$ and 0.4 (left figure), and as a function of $U$ for $L=32$ and 128 (right figure). For values of $L$ larger than $M^{-1}$ (the inverse mass gap) $\chi_{1}$ begins to saturate. For each value of $L$, we can also define the mass scale $M_{b}=L^{-1}$ defined at the location of the peak $U=U_{\mathrm{p}}$. We see that roughly $M \sim M_{b}$. (b) Fluctuations of $\chi_{1}$ in a sample of 500 vertex configurations $[x ; k]$ generated consecutively during the Monte Carlo sampling. Rare but large fluctuations seem to be the remnant of infrared divergences.

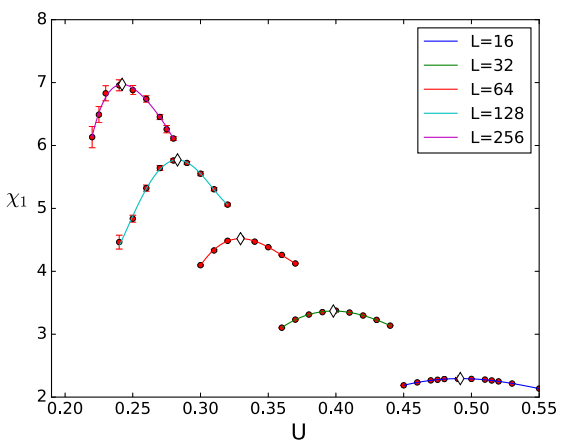

(a)
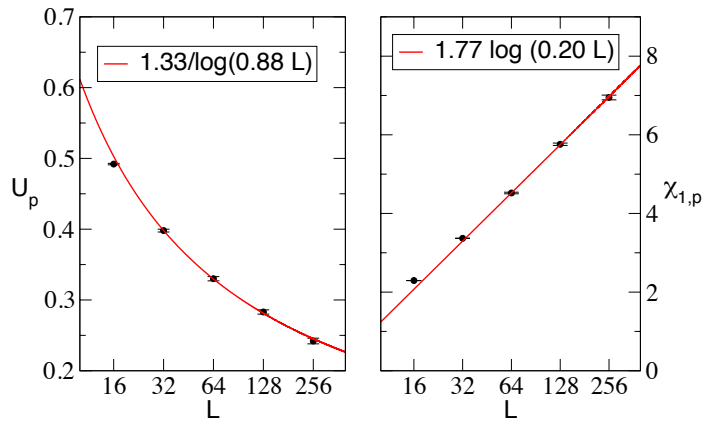

(b)

Figure 5. (a) Plot of $\chi_{1}$ as a function of $U$ for different values of $L$. The locations of the peak obtained by fitting the data to a smooth curve (quartic functions) are listed in Tab. 1. (b) Plot of $U_{\mathrm{p}}$ and $\chi_{1, \mathrm{p}}$ as a function of $L$ and the fits to Eq.(11).

fluctuations in the Monte Carlo approach. We do see such rare fluctuations in our data. For example in Fig. 4(b) we plot the fluctuations in $\chi_{1}$ during a sample of the Monte Carlo time history for $L=64$ and $U=0.4$. As can be seen in Fig. 4(a), for these parameters the susceptibility has already saturated to the value $\chi_{1} \approx 3.7$. However, as Fig. 4(b) shows there are still large but rare fluctuations in $\chi_{1}$ that are five times larger. The fact that these divergent contributions are rare compared to finite contributions cannot be easily uncovered in usual perturbation theory. 
For a quantitative analysis, instead of measuring the conventional mass gap we can define a slightly different mass scale $M_{b}$ as follows. We first note that the susceptibility $\chi_{1}$ has a peak when it is plotted as a function of $U$ for every fixed value of $L$. This behavior is clearly visible in the right figure of Fig. 4(a) where we plot $\chi_{1}$ as a function of $U$ at $L=32$ and 128. For a fixed $L$ if the peak occurs at $U=U_{\mathrm{p}}$, we define $M_{b} \equiv L^{-1}$ as the non-perturbative mass scale generated at $U=U_{\mathrm{p}}$. Comparing the left and right figures in Fig.4(a) we see that our definition of $M_{b}$ is also roughly consistent with the value of $M$ obtained using the value of $L$ where $\chi_{1}$ begins to level off. We have located the peaks at various lattice sizes accurately by fitting the data to quartic functions as shown in Fig.5(a). Table 1 gives the values of the peak obtained through such a fit at different values of $L$.

Table 1. Fit values for $\chi_{1, \mathrm{p}}$ and $U_{\mathrm{p}}$ as a function of $L$.

\begin{tabular}{|c|c|c|}
\hline$L$ & $\chi_{1, \mathrm{p}}$ & $U_{\mathrm{p}}$ \\
\hline 16 & $2.293(2)$ & $0.492(1)$ \\
32 & $3.368(5)$ & $0.398(2)$ \\
64 & $4.520(20)$ & $0.330(3)$ \\
128 & $5.760(30)$ & $0.283(3)$ \\
256 & $6.950(60)$ & $0.242(4)$ \\
\hline
\end{tabular}

If our theory is asymptotically free we expect that $M_{b}=\Lambda \exp \left(-\beta / U_{\mathrm{p}}\right)$. Further, the peak value of the susceptibility $\chi_{1, p}$ is expected to grow logarithmically in the continuum limit. Thus, for sufficiently large values of $L$ we expect

$$
\chi_{1, \mathrm{p}}=\alpha \log \left(\Lambda_{1} L\right), \quad U_{\mathrm{p}}=\frac{\beta}{\log \left(\Lambda_{2} L\right)},
$$

In Fig. 5(b) we show that our results are consistent with both these expectations. The parameters obtained from the fit to our data gives $\alpha=1.77(4), \beta=1.33(4), \Lambda_{1}=0.20(1)$ and $\Lambda_{2}=0.88(9)$. It is usually difficult to match $\beta$ with one loop perturbation theory since very large correlation lengths are needed [17]. On the other hand, qualitative exponential scaling of mass gaps as we show here, can be observed more easily [18].

\section{Conclusions}

In this work we have shown that weak coupling Feynman diagrams contain the information of a nonperturbative mass gap in an asymptotically free theory. Using a specific lattice model we first tamed the infrared divergences in the usual perturbation theory by formulating the problem in a finite volume. We then showed that the physics of the mass gap arises at sufficiently large volumes when we sample Feynman diagrams containing a finite density of interactions. The infrared divergences of the original perturbative expansion seem to be hidden in a few statistically insignificant vertex configurations. Our work suggests that a perturbative expansion organized in terms of Feynman diagrams containing a fixed density of interactions may be worth exploring. Extensions of our work to gauge theories would also be interesting, but we may have to first formulate the expansion in a gauge invariant way. 


\section{References}

[1] N.V. Prokof'ev, B.V. Svistunov, Phys. Rev. Lett. 81, 2514 (1998)

[2] N. Prokof'ev, B. Svistunov, Phys. Rev. Lett. 99, 250201 (2007)

[3] M. Boninsegni, N.V. Prokof'ev, B.V. Svistunov, Phys. Rev. E 74, 036701 (2006)

[4] N.V. Prokof'ev, B.V. Svistunov, Phys. Rev. B 77, 125101 (2008)

[5] E. Kozik, et.al., Europhys. Lett. 90, 10004 (2010)

[6] G. Cohen, E. Gull, D.R. Reichman, A.J. Millis, Phys. Rev. Lett. 115, 266802 (2015)

[7] P.V. Buividovich, PoS LATTICE2015, 293 (2016)

[8] P.V. Buividovich, A. Davody (2017), arXiv: 1705.03368

[9] A. Cherman, D. Dorigoni, G.V. Dunne, M. Unsal, Phys. Rev. Lett. 112, 021601 (2014)

[10] P. Orland, Phys. Rev. D90, 125038 (2014), 1410.2627

[11] D.J. Gross, A. Neveu, Phys. Rev. D 10, 3235 (1974)

[12] E. Witten, Nuclear Physics B 145, 110 (1978)

[13] V. Ayyar, S. Chandrasekharan (2017), arXiv: 1709.06048

[14] H. Sharatchandra, H. Thun, P. Weisz, Nucl.Phys. B192, 205 (1981)

[15] C. van den Doel, J. Smit, Nucl.Phys. B228, 122 (1983)

[16] V. Ayyar, S. Chandrasekharan, Journal of High Energy Physics 2016, 58 (2016)

[17] B.B. Beard, R.J. Birgeneau, M. Greven, U.J. Wiese, Phys. Rev. Lett. 80, 1742 (1998)

[18] M. Creutz, Phys. Rev. Lett. 45, 313 (1980) 